\title{
Impact of preoperative diagnostic TURBT on progression-free survival in patients with pathological high-grade, stage T3/T4 bladder urothelial carcinoma
}

\author{
Haichao Huang ${ }^{1, *}$, Zhenhua $\mathrm{Liu}^{2, *}$, Xin $\mathrm{Li}^{2}$, Wei $\mathrm{Li}^{1}$, Jinchun $\mathrm{Xing}^{1}$, Wei $\mathrm{Yu}^{2}$ and Jie \\ Jin $^{2}$ \\ ${ }^{1}$ Department of Urology, The First Affiliated Hospital of Xiamen University, Siming District, Xiamen, Fujian 361003, China \\ ${ }^{2}$ Department of Urology, Peking University First Hospital and Institute of Urology, Peking University, National Urological Cancer \\ Center, Xicheng District, Beijing 100034, China \\ "These authors contributed equally to this work \\ Correspondence to: Wei Yu, email: yuwei_bjmu@163.com \\ Jinchun Xing, email: xmcua_2007@163.com
}

Keywords: diagnostic TURBT, cystoscopic biopsy, progression-free survival, urothelial carcinoma, bladder

Received: March 17, $2017 \quad$ Accepted: June 28, $2017 \quad$ Published: July 11, 2017

Copyright: Huang et al. This is an open-access article distributed under the terms of the Creative Commons Attribution License 3.0 (CC BY 3.0 ), which permits unrestricted use, distribution, and reproduction in any medium, provided the original author and source are credited.

\section{ABSTRACT}

Transurethral bladder tumor resection (TURBT) reportedly increases the circulating tumor cell count in patients with urothelial carcinoma of the bladder (UCB). To determine whether diagnostic TURBT leads to poorer progression-free survival (PFS) than diagnostic cystoscopic biopsy, we retrospectively reviewed the records of 96 consecutive primary pathological high-grade, stage T3/T4 UCB patients treated with radical cystectomy (RC) between January 2009 to December 2013. Clinicopathological features were collected from the medical records. PFS was determined from KaplanMeier curves, and potential independent prognostic factors for PFS were identified based on multivariable Cox analysis. During the follow-up period (median, 29 months), 43 patients experienced tumor progression ( 40 received diagnostic TURBT, 56 received cystoscopic biopsy). Patients who received cystoscopic biopsy had better PFS than those who received diagnostic TURBT $(p=0.008)$. Additionally, diagnostic TURBT was a significant risk factor for tumor metastasis in both univariable (HR: 2.219; 95\% CI: 1.207-4.079; $p=0.010$ ) and multivariable (HR: 2.455; 95\% CI: 1.278-4.714; $p=0.007$ ) Cox analyses. The present study provides the first evidence that diagnostic TURBT before RC negatively affects PFS in patients with pathological high-grade, stage T3/T4 UCB.

\section{INTRODUCTION}

Bladder cancer is the most common malignancy of the urinary tract [1], and UCB is the most common pathological subtype. RC with pelvic lymph node dissection has been used as the standard therapy for muscle-invasive bladder cancer (MIBC), but the 5-year survival after RC for clinically localized MIBC is only approximately $50 \%$ [2]. The 5 -year overall survival and tumor-specific survival for UCB patients with extravesical stage are only $30 \%$ and $37 \%$, respectively [3-4]. The presence of circulating tumor cells (CTCs) has been confirmed to be associated with survival in several solid malignancies [5]. CTCs were detected in $17-50 \%$ of UCB patients and were significantly associated with tumor stage [6-8]. Diagnostic transurethral bladder tumor resection (TURBT) represents the standard initial, preoperative diagnostic procedure for MIBC. However, TURBT can damage the integrity of the bladder wall. Moreover, the pressure within the bladder during the process of TURBT would exceed the venous pressure, and it is impossible to completely resect the tumor for patients with extravesical stage (T3 and T4). Taken together, there is an increasing concern that TURBT may increase the risk of tumor metastasis. Recent studies revealed that TURBT may increase the count of CTCs for MIBC patients 
postoperatively [9-10]. Further studies are needed to determine whether TURBT increases the risk of metastatic diseases for patients with MIBC. Cystoscopic biopsy, another conventional initial diagnostic method for bladder cancer, seems to show less risk of metastatic events, as it causes relatively minor damage to the integrity of the bladder wall. In the present study, we evaluated whether preoperative diagnostic TURBT, compared with preoperative diagnostic cystoscopic biopsy, increased the risk of tumor progression for high-grade, stage T3/T4 UCB patients who underwent eventual RC.

\section{MATERIALS AND METHODS}

\section{Patients and data collection}

We retrospectively enrolled 96 patients with postoperative histological diagnosis as high-grade, stage T3/T4 UCB who underwent RC at two Chinese centers (Department of Urology, The First Affiliated Hospital of Xiamen University and Department of Urology, Peking University First Hospital) between January 2009 and December 2013. Patients with previous bladder tumors and other malignancies were excluded. Clinicopathological features, including age, gender, smoking history, method for obtaining preoperative histological evidence (diagnostic TURBT vs. cystoscopic biopsy), tumor size and number, tumor stage (T3 vs. T4), the presence of glandular differentiation, sarcomatoid differentiation, squamous differentiation, concomitant Carcinoma In Situ (CIS), lymphovascular invasion (LVI), prostatic urethra invasion (PUI), lymph node metastasis (LNM), post-operative adjuvant chemotherapy, and prognostic outcomes were collected. All patients received either diagnostic TURBT or diagnostic cystoscopic biopsy before the eventual RC.

\section{Treatment and follow-up}

$\mathrm{RC}$ with pelvic lymph node dissection with or without postoperative adjuvant chemotherapy was applied as the main therapy for all patients within one month after the initial diagnostic TURBT or cystoscopic biopsy. The final pathological data analyzed in this study were based on post-RC standard pathological procedures. Tumor stage was assessed according to the Union for International Cancer Control (UICC) TNM classification of malignant tumors 2002. Tumor grade was assessed according to the WHO classification of 2004. The patients were seen every 6 months after RC. The follow-up visits consisted of a physical examination and ultrasonography. Other imaging analyses, including chest radiography and abdominal $\mathrm{CT}$, were indicated if necessary. Local recurrences were defined as those occurring within the soft tissue field of exenteration, which is inside the bony pelvis. Distant recurrences were those occurring outside the pelvis [11-12].
Time to recurrence was defined as the period from the date of $\mathrm{RC}$ to the date when the first recurrence was diagnosed. Completed follow-up data were available for 86 patients.

\section{Statistical analysis}

For statistical analysis, numerical variables with $t$-test and categorical variables with chi-square test were used for comparative analyses between diagnostic TURBT and cystoscopic biopsy groups. Survival curves were plotted using the Kaplan-Meier method, and significant differences were assessed using the Log-rank test. Subsequently, Cox regression analysis was applied to identify potential prognostic factors for survival. Characteristics that showed potential significance $(p<0.1)$ were further evaluated using multivariable Cox regression model. SPSS 22.0 was used in all statistical analyses. In all tests, $p<0.05$ were considered to indicate significance. All experimental protocols were approved by Ethics Committee of Peking University First Hospital and Ethics Committee of The First Affiliated Hospital of Xiamen University. Informed consent was obtained from all subjects.

\section{RESULTS}

\section{Baseline characteristics}

A total of 96 high-grade, stage T3/T4 UCB patients were involved in our study; 27 patients were treated in the Department of Urology, The First Affiliated Hospital of Xiamen University, while the remaining 69 patients were treated in the Department of Urology, Peking University First Hospital. Based on the preoperative diagnostic methods, patients were grouped into the diagnostic cystoscopic biopsy group $(n=56)$ or the diagnostic TURBT group $(n=40)$. Analyses of these two cohorts demonstrated no statistically significant differences with regard to patient demographics, tumor number, tumor stage, the presence of glandular differentiation, sarcomatoid differentiation, squamous differentiation, concomitant CIS, LVI, PUI, LNM, or post-operative adjuvant chemotherapy status. However, there were significant differences with respect to tumor size between the two cohorts $(89.3 \%$ with larger size for the biopsy group vs. $70.0 \%$ for the diagnostic TURBT group, $p=0.017$ ) (Table 1). In addition, 37 patients received post-operative chemotherapy, among which 32 received gemcitabine/cisplatin (GC), 3 received gemcitabine/ carboplatin, and 2 received gemcitabine/ paclitaxel regimen. The clinicopathological features of samples from the two Chinese centers are described in Table 2. Compared with patients selected from Peking University First Hospital, patients selected from The First Affiliated Hospital of Xiamen University had a larger proportion of patients aged 60 years or older $(p=0.011)$. No significant difference regarding the remaining clinicopathological 
Table 1: Descriptive clinicopathologic characteristics of patients treated with radical cystectomy and bilateral lymphadenectomy for pathological high-grade, stage T3/T4 urothelial carcinoma of the bladder

\begin{tabular}{|c|c|c|c|c|}
\hline Characteristics & $\begin{array}{c}\text { Cystoscopic biopsy, n (\%) } \\
N=56\end{array}$ & 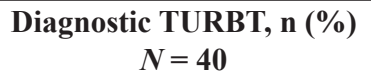 & Total & $P$ value \\
\hline Age (yr) & & & & 0.164 \\
\hline$<60$ & $20(35.7)$ & $9(22.5)$ & 29 & \\
\hline$\geq 60$ & $36(64.3)$ & $31(77.5)$ & 67 & \\
\hline Gender & & & & 0.887 \\
\hline Male & 47 (83.9) & $34(85.0)$ & 81 & \\
\hline Female & $9(16.1)$ & $6(15.0)$ & 15 & \\
\hline Smoking history & & & & 0.770 \\
\hline No & $38(67.9)$ & $26(65.0)$ & 64 & \\
\hline Yes & $18(32.1)$ & $14(35.0)$ & 32 & \\
\hline Tumor size (cm) & & & & 0.017 \\
\hline$<3$ & $6(10.7)$ & $12(30.0)$ & 18 & \\
\hline$\geq 3$ & $50(89.3)$ & $28(70.0)$ & 78 & \\
\hline Tumor number (n) & & & & 0.558 \\
\hline$<3$ & $36(64.3)$ & $28(70.0)$ & 64 & \\
\hline$\geq 3$ & $20(35.7)$ & $12(30.0)$ & 32 & \\
\hline pathologic stage & & & & 0.804 \\
\hline pT3 & $35(62.5)$ & $24(60.0)$ & 59 & \\
\hline pT4 & $21(37.5)$ & $16(40.0)$ & 37 & \\
\hline Concomitant CIS & & & & 0.426 \\
\hline Absent & $44(78.6)$ & $34(85.0)$ & 78 & \\
\hline Present & $12(21.4)$ & $6(15.0)$ & 18 & \\
\hline Squamous differentiation & & & & 0.744 \\
\hline Absent & $36(64.3)$ & $27(67.5)$ & 63 & \\
\hline Present & $20(35.7)$ & $13(32.5)$ & 33 & \\
\hline Sarcomatoid differentiation & & & & 1.000 \\
\hline Absent & $49(87.5)$ & $35(87.5)$ & 84 & \\
\hline Present & $7(12.5)$ & $5(12.5)$ & 12 & \\
\hline Glandular differentiation & & & & 0.844 \\
\hline Absent & $41(73.2)$ & $30(75.0)$ & 71 & \\
\hline Present & $15(26.8)$ & $10(25.0)$ & 25 & \\
\hline LVI & & & & 0.138 \\
\hline Absent & $25(44.6)$ & $24(60.0)$ & 59 & \\
\hline Present & $31(55.4)$ & $16(40.0)$ & 47 & \\
\hline PUI & & & & 0.102 \\
\hline Absent & $33(58.9)$ & $30(75.0)$ & 63 & \\
\hline Present & $23(41.1)$ & $10(25.0)$ & 33 & \\
\hline LN metastasis & & & & 0.129 \\
\hline Absent & $43(76.8)$ & $25(62.5)$ & 68 & \\
\hline Present & $13(23.2)$ & $15(37.5)$ & 28 & \\
\hline Postoperative adjuvant chemotherapy & & & & 0.127 \\
\hline No & $38(67.9)$ & $21(52.5)$ & 59 & \\
\hline Yes & $18(32.1)$ & $19(47.5)$ & 37 & \\
\hline Total & $56(58.3)$ & $40(41.7)$ & 96 & \\
\hline
\end{tabular}

CIS = Carcinoma In Situ; LVI = lymphovascular invasion; PUI = prostatic urethra invasion; $\mathrm{LN}=$ lymph node. 
Table 2: Descriptive clinicopathologic characteristics of patients treated with radical cystectomy and bilateral lymphadenectomy for pathological high-grade, stage T3/T4 urothelial carcinoma of the bladder in two large tertiary referral centers of China

\begin{tabular}{|c|c|c|c|c|}
\hline Characteristics & $\begin{array}{c}\text { FAHXMU, } \boldsymbol{n}(\%) \\
\quad \boldsymbol{N}=\mathbf{2 7}\end{array}$ & $\begin{array}{c}\text { PKUFH, } n(\%) \\
\quad N=69\end{array}$ & Total & $P$ value \\
\hline Preoperative method & & & & 0.565 \\
\hline biopsy & $17(63.0)$ & $39(56.5)$ & 56 & \\
\hline diagnostic TURBT & $10(37.0)$ & $30(43.5)$ & 40 & \\
\hline Age (yr) & & & & 0.011 \\
\hline$<60$ & $3(11.1)$ & $26(22.5)$ & 29 & \\
\hline$\geq 60$ & $24(88.9)$ & $43(77.5)$ & 67 & \\
\hline Gender & & & & 0.423 \\
\hline Male & $21(77.8)$ & $60(85.0)$ & 81 & \\
\hline Female & $6(22.2)$ & $9(15.0)$ & 15 & \\
\hline Smoking history & & & & 0.630 \\
\hline No & $19(70.4)$ & $45(65.2)$ & 64 & \\
\hline Yes & $8(29.6)$ & $24(34.8)$ & 32 & \\
\hline Tumor size $(\mathrm{cm})$ & & & & 0.971 \\
\hline$<3$ & $5(18.5)$ & $13(18.8)$ & 18 & \\
\hline$\geq 3$ & $22(81.5)$ & $56(81.2)$ & 78 & \\
\hline Tumor number (n) & & & & 0.630 \\
\hline$<3$ & $19(70.4)$ & $45(65.2)$ & 64 & \\
\hline$\geq 3$ & $8(29.6)$ & $24(34.8)$ & 32 & \\
\hline pathologic stage & & & & 0.782 \\
\hline pT3 & $16(59.3)$ & $43(62.3)$ & 59 & \\
\hline pT4 & $11(40.7)$ & $26(37.7)$ & 37 & \\
\hline Concomitant CIS & & & & 0.260 \\
\hline Absent & $20(74.1)$ & $58(84.1)$ & 78 & \\
\hline Present & $7(25.9)$ & $11(15.9)$ & 18 & \\
\hline Squamous differentiation & & & & 0.411 \\
\hline Absent & $16(59.3)$ & $47(68.1)$ & 63 & \\
\hline Present & $11(40.7)$ & $22(31.9)$ & 33 & \\
\hline Sarcomatoid differentiation & & & & 0.198 \\
\hline Absent & $26(96.3)$ & $58(84.1)$ & 84 & \\
\hline Present & $1(2.7)$ & $11(15.9)$ & 12 & \\
\hline Glandular differentiation & & & & 0.616 \\
\hline Absent & $19(70.4)$ & $52(75.4)$ & 71 & \\
\hline Present & $8(29.6)$ & $17(24.6)$ & 25 & \\
\hline LVI & & & & 0.207 \\
\hline Absent & $11(40.8)$ & $38(55.1)$ & 49 & \\
\hline Present & $16(59.2)$ & $31(44.9)$ & 47 & \\
\hline PUI & & & & 0.893 \\
\hline Absent & $18(66.7)$ & $45(65.2)$ & 63 & \\
\hline Present & $9(33.3)$ & $24(34.8)$ & 33 & \\
\hline LN metastasis & & & & 0.151 \\
\hline Absent & $22(81.5)$ & $46(66.7)$ & 68 & \\
\hline Present & $5(18.5)$ & $23(33.3)$ & 28 & \\
\hline
\end{tabular}


Postoperative adjuvant chemotherapy

$\begin{array}{cccc}\text { No } & 20(74.1) & 39(56.5) & 59 \\ \text { Yes } & 7(25.9) & 30(43.5) & 37 \\ \text { Total } & 27(28.1) & 69(71.9) & 96\end{array}$

FAHXMU = The First Affiliated Hospital of Xiamen University; PKUFH = Peking University First Hospital; CIS = Carcinoma In Situ; LVI = lymphovascular invasion; PUI = prostatic urethra invasion; $\mathrm{LN}=$ lymph node.

features between samples from the two centers was observed.

\section{Survival outcomes}

Completed follow-up data were available for 86 patients, with a median follow-up period of 29 months. During the follow-up period, 43 patients experienced disease progression, 18 from the preoperative diagnostic cystoscopic biopsy group and 25 from the preoperative diagnostic TURBT group. Survival data displayed in Kaplan-Meier curves demonstrated significant differences with respect to PFS between diagnostic TURBT and cystoscopic biopsy groups $(p=0.008$, Figure 1$)$. The 3 -year PFS rates for patients in the diagnostic biopsy and TURBT groups were $63.6 \%$ and $48.1 \%$, respectively.
In the univariable Cox analysis, preoperative diagnostic TURBT, smoking history, the presence of sarcomatoid differentiation, LNM, and higher pathological stage (pT4) were significantly associated with an unfavorable PFS. However, only preoperative diagnostic TURBT, smoking history, the presence of sarcomatoid differentiation and higher pathological stage (pT4) retained the significant association with survival following the multivariable analysis (Table 3 ).

\section{DISCUSSION}

Bladder cancer is the most common malignancy of the urinary tract [1]. On average, $70 \%$ of bladder tumors present as non-muscle-invasive bladder cancer (NMIBC), the remaining $30 \%$ as MIBC. For the MIBC

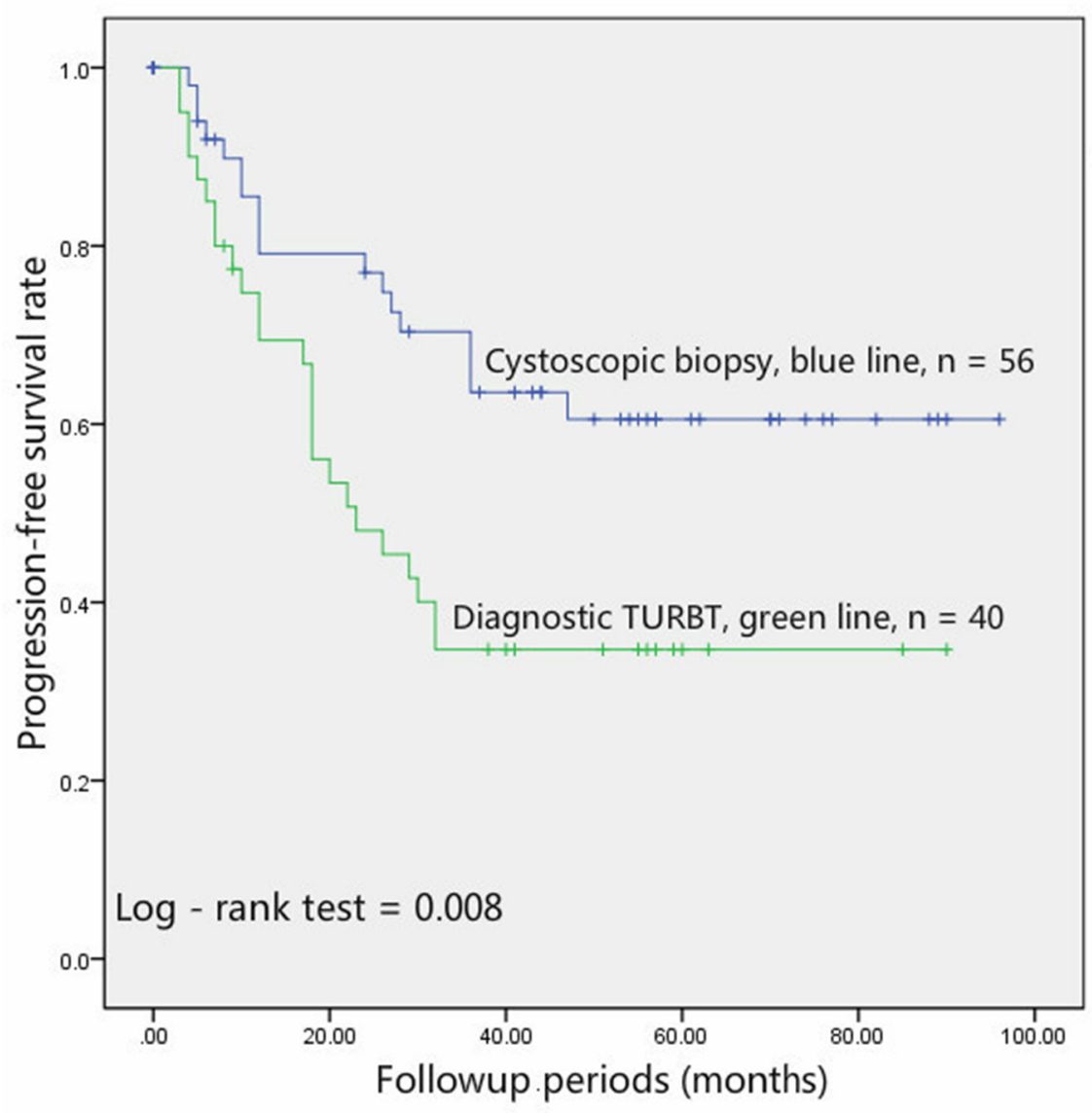

Figure 1: Patients undergoing diagnostic cystoscopic biopsy (blue line, $n=56$ ) had a significantly $(p=0.008)$ better PFS than those undergoing diagnostic TURBT (green line, $n=40$ ). 
Table 3: Univariate and multivariate Cox regression analyses for prediction of progression-free survival in 96 patients treated with radical cystectomy and bilateral lymphadenectomy for highgrade, stage T3/T4 urothelial carcinoma of the bladder

\begin{tabular}{cccc}
\hline Univariate analyses & HR & $\mathbf{9 5 \%}$ CI & $\boldsymbol{P}$ \\
\hline TURBT before RC & 2.219 & $1.207-4.079$ & 0.010 \\
Postoperative adjuvant chemotherapy (yes) & 0.792 & $0.427-1.471$ & 0.461 \\
Smoking history (+) & 1.670 & $0.912-3.055$ & 0.096 \\
Tumor size $(\geq 3 \mathrm{~cm})$ & 0.876 & $0.406-1.890$ & 0.736 \\
Tumor number $(\geq 3 \mathrm{~cm})$ & 1.160 & $0.625-2.154$ & 0.638 \\
Male gender & 1.987 & $0.710-5.563$ & 0.191 \\
Age ( $\geq$ 60 yr) & 1.416 & $0.712-2.817$ & 0.322 \\
Sarcomatoid differentiation (present) & 1.915 & $0.887-4.137$ & 0.098 \\
Squamous differentiation (present) & 0.973 & $0.507-1.866$ & 0.934 \\
Glandular differentiation (present) & 0.784 & $0.395-1.556$ & 0.486 \\
PUI (+) & 1.096 & $0.590-2.036$ & 0.771 \\
LVI (+) & 0.872 & $0.478-1.593$ & 0.656 \\
Concomitant CIS (present) & 0.686 & $0.289-1.626$ & 0.392 \\
LN metastasis (+) & 1.997 & $1.074-3.712$ & 0.029 \\
Pathologic tumor stage (T4) & 1.830 & $1.006-3.331$ & 0.048 \\
\hline Multivariate analyses & HR & $\mathbf{9 5 \%}$ CI & $\boldsymbol{P}$ \\
\hline TURBT before RC & 2.455 & $1.278-4.714$ & 0.007 \\
Smoking history (+) & 1.958 & $1.040-3.686$ & 0.037 \\
Sarcomatoid differentiation (present) & 2.965 & $1.285-6.843$ & 0.011 \\
LN metastasis (+) & 1.389 & $0.715-2.698$ & 0.332 \\
Pathologic tumor stage (T4) & 2.004 & $1.085-3.703$ & 0.026 \\
\hline
\end{tabular}

CIS = Carcinoma In Situ; LVI = lymphovascular invasion; PUI = prostatic urethra invasion; LN = lymph node.

group, LNM occurs in approximately $25 \%$ patients [6]. Moreover, although RC represents the current gold standard therapy for MIBC, distant metastasis occurs in $50 \%$ of MIBC patients within 2 years [13]. CTCs are cancer cells which originate from the primary tumor site and invade the lymphatics and systemic circulation during the initial sequences of metastatic progression [14-15]. While the majority of CTCs die in the bloodstream, a few cells eventually form new colonies at distant sites. Thus, the presence of CTCs may indicate micrometastasis, which represents an early stage of the metastatic process. In addition, CTCs, which are associated with tumor recurrence and metastasis in other solid malignancies (including, breast, rectum, and prostate cancer), have emerged as a potential candidate to explain the high progression rate of MIBC [5].

Recent studies showed that TURBT increases the count of CTCs for UCB patients, especially for the MIBC population. Engilbertsson and colleagues [9] evaluated 16 UCB patients (10 MIBC, 6 NMIBC) who underwent TURBT, from which 6 (5 MIBC, 1 NMIBC) positive inferior vena cava samples were found to have an increased number of CTCs intraoperatively. In addition, Blaschke and colleagues reported a similar result recently, where the CTC count increased postoperatively in all of the T2 high-grade UCB patients [10]. However, Antoniewicz and colleagues [16] evaluated CTC count of 51 UCB patients. In their study, no significant evidence for increased level of circulating urothelial cells in the peripheral blood after TURBT was found. Interestingly, half of their included patients had NMIBC. Taken together, MIBC patients, compared with NMIBC patients, are more likely to be affected by the TURBT. Moreover, in the systematic review and meta-analysis of Msaouel and Koutsilieris [7], CTC-negative patients compared favorably to CTC-positive patients with respect to the proportion of advanced (stage III-IV) disease. Thus, further studies are clearly required to ascertain whether diagnostic TURBT increases the risk of metastatic diseases for UCB patients with extravesical pathological stage (T3 and T4).

Both TURBT and cystoscopic biopsy are performed to obtain histologic evidence for patients with suspicion of UCB in daily clinical work. Diagnostic TURBT provides more pathological information than cystoscopic biopsy, which is of great help in pathological staging diagnosis and therapeutic decision-making. However, compared 
with cystoscopic biopsy, TURBT makes larger wounds in the bladder wall, induces higher level of pressure within the bladder, and causes some complications especially for T3-4 patients, such as bladder perforation. Moreover, diagnostic TURBT costs more in medical fees than diagnostic cystoscopic biopsy. Thus, patients who received cystoscopic biopsy as the diagnostic surgical approach before $\mathrm{RC}$ were identified as the control group, comparing the oncologic outcomes with those received diagnostic TURBT before RC and verifying the impact of diagnostic TURBT in tumor metastasis for high-grade, stage T3/T4 UCB. In addition, although CT and MRI have limited capability to detect microscopic invasion of the perivesical fat, it may be used to find $\mathrm{T} 3 \mathrm{~b}$ disease or higher with greater diagnostic accuracy [17]. Taken together, some surgeons of our centers were inclined to choose diagnostic cystoscopic biopsy for patients with clinical T3 and $\mathrm{T} 4$ stage confirmed by CT scans before eventual RC. Considering the retrospective feature of our study, whether a patient received diagnostic TURBT or cystoscopic biopsy was based on surgeon-patient decision rather than other standards. In addition, we found that there were more patients with larger tumor size in the biopsy group than in the TURBT group in our cohort ( $p=0.017)$, suggesting that tumor size was an important factor that was considered in making the surgeon-patient decision. In other words, when a larger tumor was detected, the biopsy, rather than the diagnostic TURBT, was more likely to be performed.

In the survival analysis, patients who received cystoscopic biopsy showed a longer PFS than those underwent diagnostic TURBT ( $p=0.008$, Figure 1). In addition, the univariable and multivariable Cox analysis showed that diagnostic TURBT increased the risk the tumor metastasis ( $p=0.010$ and 0.007 , respectively). Our study suggests that diagnostic TURBT may increase the risk of tumor metastasis and compromise the PFS for high-grade, stage T3/T4 UCB patients. The potential mechanisms by which diagnostic TURBT compromises the PFS of high-grade, stage T3/T4 UCB may be attributed to the fact that the process of TURBT increases the level of CTCs and induces micrometastasis. To the best of our knowledge, we are the first to provide the evidence that diagnostic TURBT, using patients underwent cystoscopic biopsy before RC as the control group, may induce a poor PFS of high-grade, stage T3/T4 UCB patients.

The current study was limited by the inherent retrospective feature, which makes it impossible to evaluate the differential impact between TURBT and biopsy in increasing the degree of CTCs count. Another limitation lies in the fact that none of the patients enrolled in the current study received neoadjuvant chemotherapy (NAC). Indeed, NAC could improve the survival of patients with MIBC. However, no molecular profiling of the tumor is now available to identify responders, which will lead to overtreatment of nonresponders. In addition, delayed cystectomy in nonresponders may compromise final outcome. Thus, even in the majority of American and European clinical practice, the utilization rate of NAC was low [18-22]. In our centers, though NAC was recommended to patients, no patient decided to undergo NAC before the $\mathrm{RC}$, which can be attributed to the concerns mentioned above. Furthermore, as we only included tumors with extravesical stage (pathological stage T3 and T4), whether diagnostic TURBT remains as a risk factor for tumor metastasis for tumors with organconfined stage (stage T1-2) is unknown. Other Limitations of our investigation include the relatively small sample size and short follow-up period. However, for tumors with this pathological stage, the majority of progression events would occur within 2 years after the RC.

\section{CONCLUSIONS}

In conclusion, our results provide the first evidence that diagnostic TURBT before RC may induce an unfavourable PFS for patients with pathological highgrade, stage T3/T4 UCB. However, further prospective, randomized studies with larger sample size and longer follow-up period are needed.

\section{Abbreviations}

TURBT = transurethral bladder tumor resection; $\mathrm{UCB}=$ urothelial carcinoma of bladder; $\mathrm{PFS}=$ progression-free survival; $\mathrm{RC}=$ radical cystectomy; $\mathrm{MIBC}$ $=$ muscle invasive bladder cancer; $\mathrm{CTCs}=$ circulating tumor cells; CIS = concomitant Carcinoma In Situ; PUI = prostatic urethra invasion; LVI = lymphovascular invasion; $\mathrm{LNM}=$ lymph node metastasis; NAC = neoadjuvant chemotherapy; NMIBC = non-muscle invasive bladder cancer.

\section{Authors' contributions}

Haichao Huang collected the data of The First Affiliated Hospital of Xiamen University, wrote the article, performed the statistic works and analyzed the data. Zhenhua Liu collected the data of Peking University First Hospital. Haichao Huang and Zhenhua Liu contributed equally to this work. Wei Yu and Jinchun Xing designed the study. Jie Jin reviewed the article and made suggestions for revision. Xin Li contributed the figures. Wei Li provided the tables. All authors approved the final manuscript as submitted and agree to be accountable for all aspects of the work.

\section{CONFLICTS OF INTEREST}

The authors declare that no conflicts of interest exists. 


\section{FINANCIAL SUPPORT}

None to declare.

\section{REFERENCES}

1. Siegel R, Ma J, Zou Z, Jemal A. Global cancer statistics, 2014. CA Cancer J Clin. 2014; 64:9-29.

2. Stein JP, Skinner DG. Radical cystectomy for invasive bladder cancer: long-term results of a standard procedure. World J Urol. 2006; 24: 296-304.

3. Gschwend JE, Dahm P, Fair WR. Disease specific survival as endpoint of outcome for bladder cancer patients following radical cystectomy. Eur Urol. 2002; 41:440-8.

4. Stein JP, Lieskovsky G, Cote R, Groshen S, Feng AC, Boyd S, Skinner E, Bochner B, Thangathurai D, Mikhail M, Raghavan D, Skinner DG. Radical cystectomy in the treatment of invasive bladder cancer: long-term results in 1054 patients. J Clin Oncol. 2001; 19:666-75.

5. Maheswaran S, Haber DA. Circulating tumor cells: a window into cancer biology and metastasis. Curr Opin Genet Dev. 2010; 20:96-9.

6. Pashos CL, Botteman MF, Laskin BL, Redaelli A. Bladder cancer: epidemiology, diagnosis, and management. Cancer pract. 2002; 10:311-22.

7. Msaouel P, Koutsilieris M. Diagnostic value of circulating tumor cell detection in bladder and urothelial cancer: systematic review and meta-analysis. BMC cancer. 2011; 11: 336 .

8. Flaig TW, Wilson S, van Bokhoven A, Varella-Garcia M, Wolfe P, Maroni P, Genova EE, Morales D, Lucia MS. Detection of circulating tumor cells in metastatic and clinically localized urothelial carcinoma. Urology. 2011; 78:863-7.

9. Engilbertsson $\mathrm{H}$, Aaltonen KE, Björnsson $\mathrm{S}$, Kristmundsson T, Patschan O, Rydén L, Gudjonsson S. Transurethral bladder tumor resection can cause seeding of cancer cells into the bloodstream. J Urol. 2015; 193:53-7.

10. Blaschke S, Koenig F, Schostak M. Hematogenous tumor cell spread following standard transurethral resection of bladder carcinoma. Eur Urol. 2016; 70:544-5.

11. Stein JP, Lieskovsky G, Cote R, Groshen S, Feng AC, Boyd S, Skinner E, Bochner B, Thangathurai D, Mikhail M, Raghavan D, Skinner DG. Radical cystectomy in the treatment of invasive bladder cancer: long-term results in 1,054 patients. J Clin Oncol. 2001; 19:666e75.
12. Mitra AP, Quinn DI, Dorff TB, Skinner EC, Schuckman AK, Miranda G, Gill IS, Daneshmand S. Factors influencing post-recurrence survival in bladder cancer following radical cystectomy. BJU Int. 2010; 109:846e64.

13. Karl A, Carroll PR, Gschwend JE, Knüchel R, Montorsi F, Stief CG, Studer UE. The impact of lymphadenectomy and lymph node metastasis on the outcomes of radical cystectomy for bladder cancer. Eur Urol. 2009; 55:826-35.

14. Mocellin S, Keilholz U, Rossi CR, Nitti D. Circulating tumor cells: the 'leukemic phase' of solid cancers. Trends Mol Med. 2006; 12:130-9.

15. Msaouel P, Pissimissis N, Halapas A, Koutsilieris M. Mechanisms of bone metastasis in prostate cancer: clinical implications. Best Pract Res Clin Endocrinol Metab. 2008; 22:341-5.

16. Antoniewicz AA, Paziewska A, Mikula M, Goryca K, Dabrowska M, Poletajew S, Borowka A, Ostrowski J. Lack of evidence for increased level of circulating urothelial cells in the peripheral blood after transurethral resection of bladder tumors. Int Urol Nephrol. 2012; 44:761-7.

17. Rajesh A, Sokhi HK, Fung R, Mulcahy KA, Bankart MJ. Bladder cancer: evaluation of staging accuracy using dynamic MRI. Clin Radiol. 2011; 66: 1140-5.

18. Porter MP, Kerrigan MC, Donato BM, Ramsey SD. Patterns of use of systemic chemotherapy for Medicare beneficiaries with urothelial bladder cancer. Urol Oncol. 2011; 29:252-8.

19. Zaid HB, Patel SG, Stimson CJ, Resnick MJ, Cookson MS, Barocas DA, Chang SS. Trends in the utilization of neoadjuvant chemotherapy in muscle-invasive bladder cancer: results from the National Cancer Database. Urology. $2014 ; 83: 75-80$.

20. Sfakianos JP, Galsky MD. Neoadjuvant chemotherapy in the management of muscle- invasive bladder cancer bridging the gap between evidence and practice. Urol Clin N Am. 2015; 42:181-7.

21. Schiffmann J, Sun M, Gandaglia G, Tian Z, Popa I, Larcher A, Meskawi M, Briganti A, McCormack M, Shariat SF, Montorsi F, Graefen M, Saad F, Karakiewicz PI. Suboptimal use of neoadjuvant chemotherapy in radical cystectomy patients: A population-based study. Can Urol Assoc J. 2016; 10:E82-6.

22. Reardon ZD, Patel SG, Zaid HB, Stimson CJ, Resnick MJ, Keegan KA, Barocas DA, Chang SS, Cookson MS. Trends in the Use of Perioperative Chemotherapy for Localized and Locally Advanced Muscle-invasive Bladder Cancer: A Sign of Changing Tides. Euro Urol. 2015; 67:165-70. 